\title{
A Multiuser TRDMA Uplink System with 2D Parallel Interference Cancellation
}

\author{
Feng Han, Member, IEEE, and K. J. Ray Liu, Fellow, IEEE
}

\begin{abstract}
The concept of Time-Reversal Division Multiple Access (TRDMA) has recently been proposed as a promising medium access technology for wireless broadband communications. Previous work on TRDMA mainly focuses on the multi-user downlink system. In this paper, we first introduce a TRDMAbased multi-user uplink architecture and then propose a 2D parallel interference cancellation scheme to enhance system performance. The TRDMA uplink architecture keeps the cost of endusers at a minimum level, and reuses the processing power at the base station that has already been made available for the downlink. The proposed $2 \mathrm{D}$ parallel interference cancellation scheme utilizes tentative decisions of detected symbols to effectively cancel both inter-symbol interference and inter-user interference, which significantly improve the bit-error-rate (BER) performance in high SNR regime. To further improve BER performance, a multi-stage processing can be implemented by cascading multiple stages of the proposed $2 \mathrm{D}$ interference cancellation, with a total delay that increases linearly with the number of stages, but independent of the number of users. The BER performance of the single-stage cancellation is analyzed, and the approximated theoretical result well matches the simulation results. Simulations are provided for up-to 3 stages of interference cancellation and compared with the basic TRDMA system without interference cancellation.
\end{abstract} tion.

Index Terms-Time reversal, TRDMA, interference cancella-

\section{INTRODUCTION}

$\mathbf{V}$ ERY recently, the concept of time reversal division multiple access (TRDMA) was introduced as a novel multi-user media access scheme for broadband communication systems [1]. The broadband communications over channels with large delay spread can be very challenging due to the severe inter-symbol interference (ISI). Conventionally, the multi-carrier technique (like OFDM/OFDMA) is used due to its high link spectrum efficiency, however the high peak-toaverage-power ratio (PAPR) of the OFDM waveform puts high demand in the system design and suffers from poor power efficiency [2]-[5]. Leveraging the unique temporal and spatial focusing effects of the time reversal (TR) phenomenon, the TRDMA provides a cost-effective single-carrier alternative with high power efficiency for broadband multiuser communications [6]-[8]. A comparative study between

Manuscript received August 31, 2013; revised December 18, 2013. The editor coordinating the review of this paper and approving it for publication was E. Au.

F. Han is currently with Qualcomm Inc., San Diego, CA, 92121, USA. He was with the Department of Electrical and Computer Engineering, University of Maryland, and Origin Wireless Communications Inc., College Park, MD, 20742 USA (e-mail: hanf@ieee.org).

K. J. R. Liu is with the Department of Electrical and Computer Engineering, University of Maryland, and Origin Wireless Communications Inc., College Park, MD, 20742 USA (e-mail: kjrliu@umd.edu).

Digital Object Identifier 10.1109/TCOMM.2014.013114.130632
TRDMA and OFDM/OFDMA was made in [8], where the authors compared the two broadband technologies in terms of complexity and performance. The TRDMA scheme uses the multi-path channel profile associated with each user's location as a location-specific signature for the user. In essence, each path of the multi-path channel is treated as a virtual antenna in the TRDMA, which collectively results in very high-resolution spatial focusing with "pin-point" accuracy. Meanwhile, the temporal focusing effect effectively suppresses ISI which significantly simplifies the terminal user's complexity and gives rise to higher-speed data transmission.

The authors in paper [1] focused on a broadband multiuser downlink system based on the TRDMA concept. In such a TRDMA downlink system, the base station (BS) transmits multiple simultaneous data streams to different users, each of which is associated with a unique multi-path profile of its channel in rich-scattering environments. The TRDMA downlink scheme exploits the spatial degrees of freedom of the environment, and focuses the useful signal power only at the intended locations. The time reversal mirrors (TRMs) [9], [10] at the BS first time-reverse ${ }^{1}$ the channel impulse response (CIR) of each user's channel as the user's signature waveform, and then embed these signatures into the corresponding data streams. The transmitted signal from the BS in the TRDMA downlink is a mixed signal consisting of all the users' data. When such a combined signal propagates to a certain user through the corresponding multi-path channel, a massive number of co-phased multi-path signals will automatically resonate at this user's location, resulting in the spatial focusing of the power of the useful signal component that carries this user's data.

Within the TRDMA framework, more sophisticated signature waveforms than the basic TR-waveform can be derived based on the multi-path channel responses to further improve the performance of the TRDMA downlink system, when additional computational complexity is affordable at the BS [11]. One very desirable feature of the TRDMA downlink scheme proposed in [1] is that most of the complexity can be shifted to the BS side, facilitating the very low complexity at the end-users.

In line with the same design philosophy of minimizing the complexity of the end-users, a TRDMA based uplink scheme can be developed. As one will see in this paper, the proposed TRDMA uplink scheme shares a strong duality in the mathematical structure with the downlink without increasing the complexity of the end-users. And as such, a virtual spatial

\footnotetext{
${ }^{1}$ The operation of time reversal is to rearrange the received waveform reversely over time.
} 
focusing effect (compared with the physical spatial focusing in the downlink) can be observed in the user's signature domain at the BS. Similar to that of the downlink scheme, the virtual spatial focusing effect enables the BS to use the user's TR signature waveform to extract the useful component out of the combined received signals, allowing multiple users accessing the BS simultaneously. The proposed TRDMA uplink scheme, along with the TRDMA downlink, complements a unique asymmetric architecture between the BS and the end-users for two-way communications. Such an asymmetric architecture facilitates the very low-cost end-user devices, and reuses the processing power and channel knowledge at the BS for both the downlink and uplink.

To further enhance the system performance of the TRDMA uplink, we also propose a 2-dimensional (2D) parallel interference cancellation scheme by exploring the signal structure of both the inter-symbol interference (time dimension) and the inter-user interference (IUI) (user dimension). Compared with the extensively-researched multi-user detection (MUD) techniques proposed for the CDMA systems in the existing literature [12]-[19], the 2D parallel interference cancellation scheme proposed for TRDMA system is distinguished in the following two aspects:

- Difference between TRDMA system and CDMA system: In this paper, we develop a 2D parallel interference cancellation scheme for the TRDMA system that uses the multi-path channel responses as users' signatures, as opposed to the spreading codes in the CDMA system. The proposed 2D interference cancellation is more desirable and challenging for the TRDMA system due to: First, the multi-path signature waveforms are not naturally orthogonal, thus more severe IUI is expected in the TRDMA system than in the CDMA system; Second, unlike the CDMA system, the TRDMA system allows overlaps between two adjacent symbols' signature waveforms to boost system throughput, which gives rise to ISI that is challenging to mitigate by using channel equalization alone.

- 2D cancellation versus 1D cancellation: Most existing MUD schemes for the CDMA system aim to cancel only the interference caused by the concurrent transmissions of multiple users, and leave the ISI to be dealt with by channel equalizers (such as Rake receivers, linear MMSE receivers). In the proposed $2 \mathrm{D}$ parallel interference cancellation scheme, the structures of both IUI and ISI are explored and utilized to effectively cancel the interference in both dimensions. Particularly, the cancellation of the ISI in the TRDMA system relieves the burden of the channel equalizers, and meanwhile affords a larger extent of waveform-overlapping to support a higher system throughput.

In this paper, we introduce a multi-user uplink scheme based on the concept of TRDMA and propose a 2D parallel interference cancellation technique for the TRDMA uplink system. Bearing a strong duality with the TRDMA downlink, the introduced TRDMA uplink scheme reuses the processing power at the BS that has already been made available for the downlink, and keeps the complexity of the terminal users

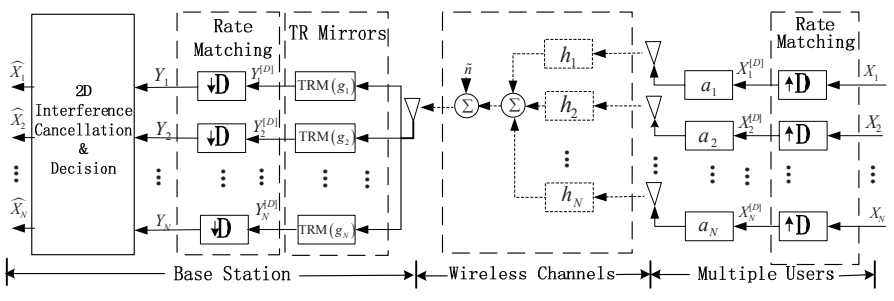

Fig. 1. The diagram of TRDMA multi-user uplink system.

at a minimal level. The proposed 2D parallel interference cancellation scheme utilizes the tentative decisions of detected symbols to effectively cancel both the ISI and IUI at the BS. To further improve the BER performance, a multi-stage processing can be performed by cascading multiple stages of the cancellation, with a total delay that increases linearly with the number of stages, but independent of the number of users. The BER performance of the single-stage cancellation is analyzed, and the approximated theoretical result is well consistent with simulation results. More simulations are provided for up-to 3 stages of interference cancellation and compared with the basic TRDMA system without interference cancellation.

The rest of this paper is organized as follows: In Section II, we introduce the TRDMA multi-user uplink scheme. In Section III, we present the proposed 2D parallel interference cancellation technique for the TRDMA uplink system, and then generalize it to a multi-stage scheme. In Section IV, the theoretical analysis is given on the BER performance of the single-stage cancellation. Section V shows the simulation results. Finally, conclusions are made in Section VI.

\section{TRDMA UPLINK SYSTEM}

Consider a multi-user broadband communications system that consists of a BS and $N$ users in the multi-path environment. In this paper, we assume that all the users operate over the same frequency band and use TRDMA to separate from one another.

Fig. 1 shows the block diagram of the TRDMA uplink scheme. As shown in Fig. 1, $N$ users simultaneously transmit independent bit streams to the BS. In this paper, we assume that the Binary Phase-Shift Keying (BPSK) modulation is used $^{2}$, and thus the polarity of the modulated symbols $\left\{X_{i}[k] \in\{-1,+1\}\right\}$ carries the binary information for User $i$.

For any given User $i$ in the uplink network, the channel $h_{i}$ between the BS and User $i$ is a multi-path channel characterized by a unique discrete-time ${ }^{3}$ channel impulse response (CIR):

$$
h_{i}[k]=\sum_{l=0}^{L-1} h_{i, l} \delta[k-l],
$$

\footnotetext{
${ }^{2}$ In this paper, BPSK is assumed for the analytical tractability. However, the proposed interference cancellation scheme can be readily generalized to accommodate other modulations such as QPSK and QAM, by simply adjusting the decision rule of the tentative decision to reflect the modulation used.

${ }^{3}$ The discrete-time channel impulse response is a sampled version of the continuous-time channel impulse response, as a result of the use of the analogto-digital convertor (ADC).
} 
where $h_{i, l} \in \mathbb{R}$ is the $l$-th tap of the CIR with length $L$, and $\delta[\cdot]$ is the Dirac delta function. We assume that the channels are quasi-static and reciprocal, which can be acquired at the BS through a channel probing phase [1], [6]. During the channel probing phase, each user takes turns to send an impulse signal ${ }^{4}$ to the BS so that the channel impulse response (CIR) $\left\{h_{i}[k]\right\}$ of each user's link can be recorded by the TRM at the BS. Upon recording the CIR, the TRM will reverse the recorded waveform in the time-domain and normalize it as the unique signature waveform of User $i[1]$. The time-reversed waveform of User $i$ will be used at the BS to extract the desired signal from a combination of the multiple access signals that are mixed in the air. Specifically, the time-reversed signature waveform of User $i$ can be written as

$$
g_{i}[k]=h_{i}[L-1-k] / \sqrt{\sum_{l=0}^{L-1}\left|h_{i}[l]\right|^{2}} .
$$

After the channel probing phase, the users can start to transmit the statistically independent messages $\left\{X_{1}[k], X_{2}[k], \cdots, X_{N}[k]\right\}$ to the BS through the multi-path channels. A rate back-off factor $D$ is introduced to match the symbol rate (signal bandwidth) with the system's sampling rate (channel bandwidth) ${ }^{5}$. For any user $U_{i}, i \in\{1,2, \ldots, N\}$, the rate matching process is performed by up-sampling the sequence of modulated symbols $\left\{X_{i}[k]\right\}$ by a factor $D$, as shown in Fig. 1. The up-sampled sequence of modulated symbols for User $i$ can be expressed as

$$
X_{i}^{[D]}[k]=\left\{\begin{array}{cl}
X_{i}[k / D], & \text { if } k \bmod D=0 \\
0, & \text { if } k \bmod D \neq 0
\end{array}\right.
$$

The scaling factors $a_{i}$, for $i \in\{1,2, \ldots, N\}$ in Fig. 1 are used to implement the transmit power control, whose values are assumed to be instructed by the BS through the feedback/control channel. After multiplying with the scaling factor, the sequence of $a_{i} X_{i}^{[D]}[k]$ for all $i \in\{1,2, \ldots, N\}$, is transmitted through the corresponding multi-path channel $\left\{h_{i}[k]\right\}$.

When the sequence $\left\{a_{i} X_{i}^{[D]}[k]\right\}$ propagates through its wireless channel $\left\{h_{i}[k]\right\}$, the convolution between $\left\{a_{i} X_{i}^{[D]}[k]\right\}$ and the CIR $\left\{h_{i}[k]\right\}$ is automatically taken as the channel output for User $i$. Then, all of the channel outputs for the $N$ users are mixed together in the air plus the additive white Gaussian noise (AWGN) $\tilde{n}[k]$ at the BS with zero mean and variance $\sigma_{N}^{2}$, as illustrated in Fig. 1. Consequently, the mixed signal received at the BS can be written as

$$
S[k]=\sum_{i=1}^{N} a_{i}\left(h_{i} * X_{i}^{[D]}\right)[k]+\tilde{n}[k] .
$$

Upon receiving the mixed signal as shown in (4), the BS passes this mixed signal through a bank of $N$ TRMs, each of which performs the convolution between its input signal

\footnotetext{
${ }^{4}$ Although it is difficult to send an ideal impulse in practice which would require infinite bandwidth, a modified raise-cosine signal can be a good candidate for limited bandwidth for this purpose [6].

${ }^{5}$ In general, the rate back-off factor $D$ can be chosen as any positive integer number. In some applications like Ultra-Wide Band (UWB), $D$ is typically chosen to be greater than 1 to match the symbol rate to the higher system's sampling rate.
}

$\{S[k]\}$ and the user's signature waveform $\left\{g_{i}[k]\right\}$. Such a convolution using the signature waveform exacts the useful signal component and suppresses the signals of other users. As the output of the $i$-th TRM, the convolution of $\{S[k]\}$ and the signature of User $i\left\{g_{i}[k]\right\}$ can be represented as

$$
\begin{aligned}
Y_{i}^{[D]}[k] & =\sum_{j=1}^{N} a_{j}\left(g_{i} * h_{j} * X_{j}^{[D]}\right)[k]+\left(g_{i} * \tilde{n}\right)[k] \\
& =\sum_{j=1}^{N} \sum_{l=0}^{2 L-2} a_{j}\left(g_{i} * h_{j}\right)[l] X_{j}^{[D]}[k-l]+\left(g_{i} * \tilde{n}\right)[k],
\end{aligned}
$$

in which the highest gain for User $i$ 's symbol is achieved at the temporal focusing time $l=L-1$, with

$$
\left(g_{i} * h_{i}\right)[L-1]=\sqrt{\sum_{l=0}^{L-1}\left|h_{i}[l]\right|^{2}} .
$$

Examining the equation (5) and the received signal at the terminal users in the downlink [1], the same mathematical structure can be found by switching the roles of the signature waveforms $\left\{g_{i}\right\}$ s and the CIRs $h_{i}$ s in the convolution (and ignoring the scaling factor $a_{i}$ and noise term.) Therefore, mathematically ${ }^{6}$, a virtual spatial focusing effect as observed in the downlink can be seen in the user's signature domain of the proposed uplink scheme. Such a virtual spatial focusing effect is used to separate the useful signal from the signals from others. Then the rate matching is performed by downsampling (with the same factor $D$ ) the TRMs' output signal to recover the original symbol rate of the modulated symbols of each user.

After the rate matching, the down-sampled TRM output $Y_{i}[k]$ can be obtained as ${ }^{7}$

$Y_{i}[k]=\sum_{j=1}^{N} \sum_{l=-\left\lfloor\frac{L-1}{D}\right\rfloor}^{\left\lfloor\frac{L-1}{D}\right\rfloor} a_{j}\left(g_{i} * h_{j}\right)[L-1+D l] X_{j}[k-l]+n_{i}[k]$,

where the colored noise $n_{i}[k]=\sum_{l=0}^{L-1} g_{i}[l] \tilde{n}[D k-l]=$ $\underline{g}_{i} \underline{\tilde{n}}[k]$ with $\underline{g}_{i} \triangleq\left[g_{i}[0], g_{i}[1], \cdots, g_{i}[L-1]\right]$ and $\underline{\tilde{n}}[k] \triangleq$ $[\tilde{n}[k], \tilde{n}[k-1], \ldots, \tilde{n}[k-L+1]]^{T}$. Note that the colored noise $\left\{n_{i}[k]\right\}$ is still a Gaussian random variable with zero mean and the same variance $\sigma_{N}^{2}$, since $\left\{g_{i}\right\}$ is a normalized waveform as shown in (2).

Decomposing the signal shown in (7), we have the following

${ }^{6}$ Unlike the physical spatial focusing effect observed in the downlink in which the useful signal power is concentrated at different physical locations, in the uplink, the signal power concentration in the users' signature waveform space is achieved mathematically at the BS.

${ }^{7}$ More rigorously, here $Y_{i}[k]=Y_{i}^{[D]}[L-1+D k]$ which aligns the highest temporal focusing gain $\left(g_{i} * h_{i}\right)[L-1]$ in $Y_{i}[k]$ with the transmitted symbol $X_{i}[k]$ in time for ease of simple notation. 
components as

$$
\begin{aligned}
Y_{i}[k] & =a_{i}\left(g_{i} * h_{i}\right)[L-1] X_{i}[k] \\
& +a_{i} \sum_{\substack{l=-\left\lfloor\frac{L-1}{D}\right\rfloor \\
l \neq 0}}^{\left\lfloor\frac{L-1}{D}\right\rfloor}\left(g_{i} * h_{i}\right)[L-1+D l] X_{i}[k-l] \\
& +\sum_{\substack{j=1 \\
j \neq i}}^{N} a_{j} \sum_{\substack{L=-\left\lfloor\frac{L-1}{D}\right\rfloor\\
}}^{\left\lfloor n_{i}[k] .\right.}
\end{aligned}
$$

The basic TRDMA uplink scheme uses the signal $Y_{i}[k]$ in (7) to estimate the transmitted symbol $X_{i}[k]$. A very simple decision rule can be derived to implement the maximumlikelihood estimation (MLE) for the BPSK symbols $X_{i}[k] \in$ $\{+1,-1\}$ for $i=1,2, \ldots, N$. By the central limit theorem, we model the total interference term (including the ISI and IUI) as a Gaussian random variable with zero mean ${ }^{8}$ and variance

$$
\begin{aligned}
\sigma_{I, i}^{2} & =\left|a_{i}\right|^{2} \sum_{\substack{l=-\left\lfloor\frac{L-1}{D}\right\rfloor \\
l \neq 0}}^{\left\lfloor\frac{L-1}{D}\right\rfloor}\left|\left(g_{i} * h_{i}\right)[L-1+D l]\right|^{2} \\
& +\sum_{\substack{j=1 \\
j \neq i}}^{N}\left|a_{j}\right|^{2} \sum_{l=-\left\lfloor\frac{L-1}{D}\right\rfloor}^{\left\lfloor\frac{L-1}{D}\right\rfloor}\left|\left(g_{i} * h_{j}\right)[L-1+D l]\right|^{2} .
\end{aligned}
$$

The likelihood ratio can be derived as

$$
\begin{aligned}
\Lambda\left(Y_{i}[k]\right) & =\frac{f_{i}\left[Y_{i}[k] \mid X_{i}[k]=1\right]}{f_{i}\left[Y_{i}[k] \mid X_{i}[k]=-1\right]} \\
& =\exp \left(\frac{2 a_{i}\left(g_{i} * h_{i}\right)[L-1]}{\left(\sigma_{I, i}^{2}+\sigma_{N}^{2}\right)} Y_{i}[k]\right),
\end{aligned}
$$

where $f_{i}(y \mid x)$ is the conditional probability density function (pdf) of $Y_{i}[k]$ given that $X_{i}[k]=x$ is transmitted. The simple form of likelihood ratio shown in (9) leads to a simple decision rule for the MLE, specifically,

$$
\hat{X}_{i}^{(0)}[k]=\operatorname{sgn}\left(Y_{i}[k]\right)= \begin{cases}+1, & \text { if } Y_{i}[k] \geqslant 0 \\ -1, & \text { if } Y_{i}[k]<0\end{cases}
$$

In (10), the superscript " $(0)$ " of $\hat{X}_{i}^{(0)}[k]$ indicates the initial stage of the interference cancellation procedure. Such a notation will be useful in the following discussion of multi-stage interference cancellation schemes.

The error probability of the estimator shown in (10) can be calculated based on the Gaussian approximations of the interference as follows

$$
P_{e r r}^{(0)}(i)=Q\left(\sqrt{\frac{\left|a_{i}\right|^{2} \sum_{l=0}^{L-1}\left|h_{i}[l]\right|^{2}}{\sigma_{I, i}^{2}+\sigma_{N}^{2}}}\right)=Q\left(\sqrt{S I N R_{i}^{(0)}}\right),
$$

where $S I N R_{i}^{(0)}$ is the Signal-to-Interference-plus-Noise Ratio (SINR) for User $i$ at the initial stage. From (11), one can see

\footnotetext{
${ }^{8}$ This is because the interference term is a linear combination of the zeromean binary symmetric symbols $X_{i}[k] \in\{+1,-1\}$.
}

that the error probability decreases with the achieved SINR, i.e. the quality of the signal before the final decision.

In the following sections, we discuss the 2D interference cancellation scheme which uses the estimated symbols to effectively cancel both the ISI and IUI, and significantly improves the performance.

\section{2-DimENSIONAL PARALLEL INTERFERENCE CANCEllation}

In this part, we describe the proposed 2D parallel interference cancellation scheme. We first introduce the singlestage scheme which serves as a building block of the more complicated multi-stage scheme, as will be introduced in the later part of this section.

The TRDMA system is an interference-limited system, especially in the high signal-to-noise ratio (SNR) regime. Fortunately, unlike the random noise, the interference terms shown in (8) have their own structure, which can be explored to further improve the BER performances. Since the CIRs have been obtained at the BS during the channel probing phase $^{9}$, the interference terms in (8) can be reconstructed if the relevant transmitted symbols are known. In our interference cancellation scheme, the estimated symbols from the previous stage $^{10}$ are used to approximate the interference terms in the current stage. Unlike the existing multiuser detection scheme in CDMA systems, the interference cancellation of the TRDMA system is complicated by the fact that the interference consists of two parts belonging to two different dimensions: the ISI is due to the multi-path effect of broadband channels, which is in the time domain; the IUI is caused by the simultaneous transmission of multiple users, which is in the user's signature domain. The proposed 2D parallel interference cancellation scheme for the TRDMA uplink system targets at the interference in both of the two dimensions, by exploiting the structure of interference in both dimensions.

\section{A. Tentative Decision Vector}

Due to the unique structure of TR waveform, each received symbol suffers the interference caused by those symbols transmitted before and after this symbol. According to (8), in order to ideally cancel the interference for User $i$ 's symbol $X_{i}[k]$, one has to know all the other users' transmitted symbols from time $\left(k-\left\lfloor\frac{L-1}{D}\right\rfloor\right)$ to $\left(k+\left\lfloor\frac{L-1}{D}\right\rfloor\right)$ for the IUI, i.e. $\left[X_{j}\left[k+\left\lfloor\frac{L-1}{D}\right\rfloor\right], X_{j}\left[k+\left\lfloor\frac{L-1}{D}\right\rfloor-1\right], \ldots, X_{j}\left[k-\left\lfloor\frac{L-1}{D}\right\rfloor\right]\right]$, for $j \neq i$; and User $i$ 's own transmitted symbols from time $\left(k-\left\lfloor\frac{L-1}{D}\right\rfloor\right)$ to $(k-1)$ and from $(k+1)$ to $\left(k+\left\lfloor\frac{L-1}{D}\right\rfloor\right)$ for the ISI, i.e., $\left[X_{i}\left[k+\left\lfloor\frac{L-1}{D}\right\rfloor\right], X_{i}\left[k+\left\lfloor\frac{L-1}{D}\right\rfloor-1\right], \ldots, X_{i}[k-\right.$ $\left.1], X_{i}[k+1], \ldots, X_{i}\left[k-\left\lfloor\frac{L-1}{D}\right\rfloor\right]\right]$.

In reality, tentative decisions are made in attempt to estimate these symbols. To simplify the notation in the sequel, define the vector $\underline{\hat{X}}_{j}^{(0)}[k]$ for all $j \in\{1,2, \ldots, N\}$, as the stage- 0 tentative decision vector for User $j$, as shown in (12).

Since the stage- 0 tentative decisions for User $j$ solely depend on this user's own TRM output, the tentative decision

\footnotetext{
${ }^{9}$ Note that the coefficients $\left\{a_{i}\right\}$ are in fact determined by the BS and sent to users.

${ }^{10}$ The basic TRDMA system is considered as the initial ( 0 -th) stage of the interference cancellation.
} 


$$
\begin{aligned}
& \underline{\hat{X}}_{j}^{(0)}[k] \triangleq\left[\hat{X}_{j}^{(0)}\left[k+\left\lfloor\frac{L-1}{D}\right\rfloor\right], \hat{X}_{j}^{(0)}\left[k+\left\lfloor\frac{L-1}{D}\right\rfloor-1\right], \ldots, \hat{X}_{j}^{(0)}\left[k-\left\lfloor\frac{L-1}{D}\right\rfloor\right]\right]^{T} \\
&=\left[\operatorname{sgn}\left(Y_{j}\left[k+\left\lfloor\frac{L-1}{D}\right\rfloor\right]\right), \operatorname{sgn}\left(Y_{j}\left[k+\left\lfloor\frac{L-1}{D}\right\rfloor-1\right]\right), \ldots, \operatorname{sgn}\left(Y_{j}\left[k-\left\lfloor\frac{L-1}{D}\right\rfloor\right]\right)\right]^{T} . \\
& \mathbf{U}_{i, j} \triangleq\left[\left(g_{i} * h_{j}\right)\left[L-1-D\left\lfloor\frac{L-1}{D}\right\rfloor\right],\left(g_{i} * h_{j}\right)\left[L-1-D\left(\left\lfloor\frac{L-1}{D}\right\rfloor-1\right)\right], \ldots,\left(g_{i} * h_{j}\right)\left[L-1+D\left\lfloor\frac{L-1}{D}\right\rfloor\right]\right],
\end{aligned}
$$

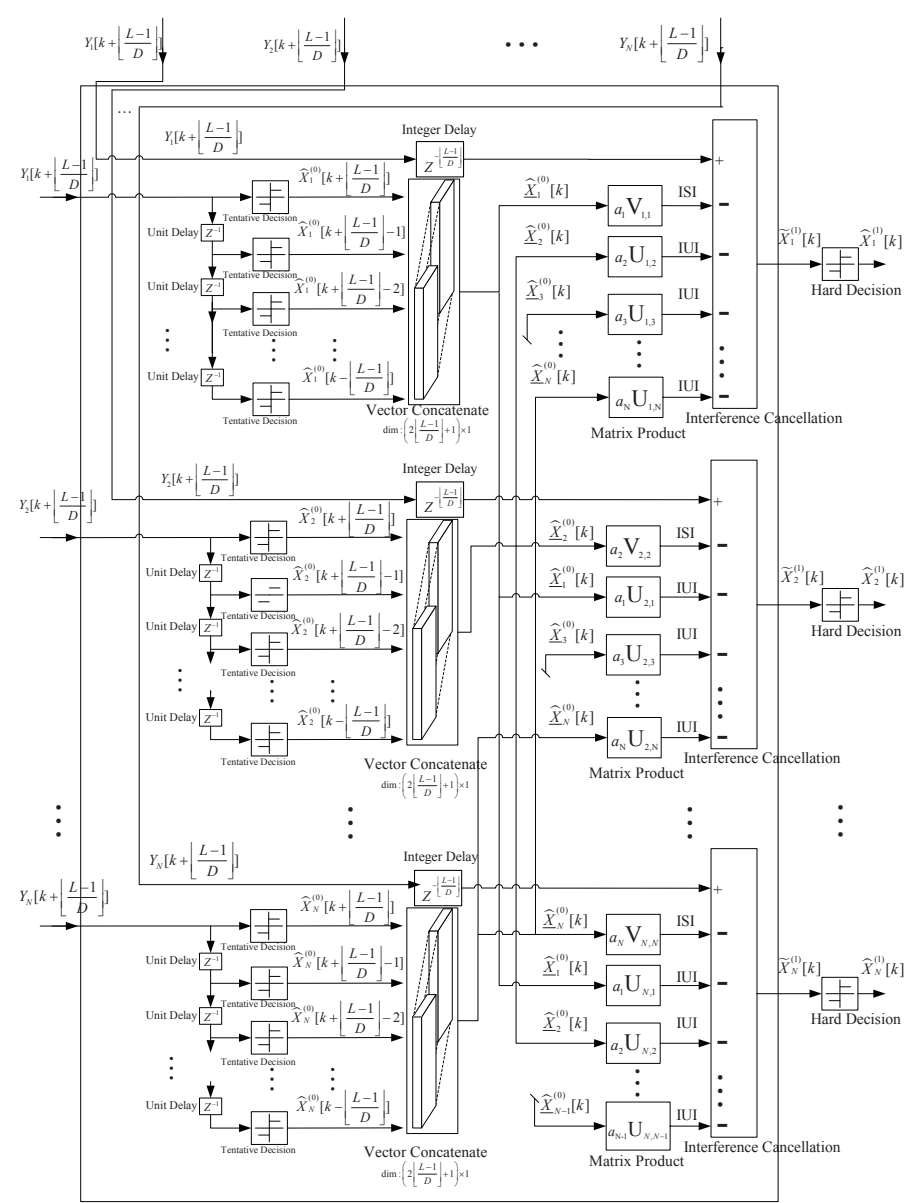

Fig. 2. The diagram of the single-stage 2D parallel interference cancellation.

vectors can be obtained in parallel for all the users in the proposed 2D parallel interference cancellation scheme.

\section{B. Approximated Interference Reconstruction}

Based on the tentative decisions of the transmitted symbols, the approximated interference terms in (8) can be reconstructed by looking at the structure of the ISI and IUI. In doing so, we first define the row vector $\mathbf{U}_{i, j}$ for $\forall i, j \in$ $\{1,2, \ldots, N\}$ as shown in (13), so that the ISI canceler vector and the IUI canceler vector can be easily represented as follows:

- ISI Canceler Vector: Considering the ISI to $X_{i}[k]$ as a linear combination of User $i$ 's own symbols, define the ISI canceler vector $\mathbf{V}_{i, i}$ for User $i$ to be

$$
\mathbf{V}_{i, i} \triangleq \mathbf{U}_{i, i} \mathcal{D}(\underline{\mathbf{1}}, 0, \underline{\mathbf{1}}),
$$

where $\mathcal{D}(\underline{z})$ is a diagonal matrix whose diagonal elements are listed by $\underline{z}$, and $\underline{\mathbf{1}} \triangleq\{1,1, \ldots, 1\}$ of length $\left\lfloor\frac{L-1}{D}\right\rfloor$. As a result, the approximated ISI term for User $i$ 's symbol $X_{i}[k]$ can be written in a compact form as the product of the ISI canceler vector $\mathbf{V}_{i, i}$ and the tentative decision vector $\underline{\hat{X}}_{i}^{(0)}[k]$ shown as follows

$$
\begin{aligned}
I S I & =a_{i} \sum_{\substack{l=-\left\lfloor\frac{L-1}{D}\right\rfloor \\
l \neq 0}}^{\left\lfloor\frac{L-1}{D}\right\rfloor}\left(g_{i} * h_{i}\right)[L-1+D l] \hat{X}_{j}[k-l] \\
& =a_{i} \mathbf{V}_{i, i} \underline{\hat{X}}_{i}^{(0)}[k] .
\end{aligned}
$$

- IUI Canceler Vector: Similarly, we define the IUI canceler vector for the IUI caused by User $j$ to User $i$ as $\mathbf{U}_{i, j}$, so that the estimated IUI term to be canceled for User $i$ 's symbol $X_{i}[k]$ can be obtained as

$$
\begin{aligned}
I U I & =\sum_{\substack{j=1 \\
j \neq i}}^{N} a_{j} \sum_{l=-\left\lfloor\frac{L-1}{D}\right\rfloor}^{\left\lfloor\frac{L-1}{D}\right\rfloor}\left(g_{i} * h_{j}\right)[L-1+D l] \hat{X}_{j}[k-l] \\
& =\sum_{\substack{j=1 \\
j \neq i}}^{N} a_{j} \mathbf{U}_{i, j} \underline{\hat{X}}_{j}^{(0)}[k] .
\end{aligned}
$$

\section{Single-stage 2D Interference Cancellation}

The single-stage 2D parallel interference cancellation scheme is shown in Fig. 2. From Fig. 2, one can see that the input signals for all the $N$ users are first buffered by delay chains of length $2\left\lfloor\frac{L-1}{D}\right\rfloor$. Then tentative hard decisions are made in parallel for each user to obtain the vector $\underline{\hat{X}}_{i}^{(0)}[k]$ for $\forall i \in\{1,2, \ldots, N\}$ using the decision rule (10). Upon obtaining the tentative decision vectors $\underline{\hat{X}}_{i}^{(0)}[k]$ for all $i \in\{1,2, \ldots, N\}$, the interference terms are reconstructed and then subtracted from the signal $Y_{i}[k]$ with the ISI and IUI canceler vectors.

Similar to the definition in (12), denoting $\underline{X}_{j}[k]$ as (17), we can rewrite (8) in a more compact form as shown in (18). After the interference cancellation, the resulting soft-bit $\tilde{X}_{i}^{(1)}[k]$ can be written as (19). A hard decision $\hat{X}_{i}^{(1)}[k]=\operatorname{sgn}\left(\tilde{X}_{i}^{(1)}[k]\right)$ can be made based on $\tilde{X}_{i}^{(1)}[k]$ to achieve a more refined estimation for the transmitted symbol.

\section{The Multi-stage Scheme}

To further improve the detection performance, a multi-stage processing can be performed by cascading multiple stages of 


$$
\begin{gathered}
\underline{X}_{j}[k] \triangleq\left[X_{j}\left[k+\left\lfloor\frac{L-1}{D}\right\rfloor\right], X_{j}\left[k+\left\lfloor\frac{L-1}{D}\right\rfloor-1\right], \ldots, X_{j}\left[k-\left\lfloor\frac{L-1}{D}\right\rfloor\right]\right]^{T} \\
Y_{i}[k]=a_{i} \sqrt{\sum_{l=0}^{L-1}\left|h_{i}[l]\right|^{2} X_{i}[k]+a_{i} \mathbf{V}_{i, i} \underline{X}_{i}[k]+\sum_{\substack{j=1 \\
j \neq i}}^{N} a_{j} \mathbf{U}_{i, j} \underline{X}_{j}[k]+n_{i}[k]} \\
\tilde{X}_{i}^{(1)}[k]=a_{i} \sqrt{\sum_{l=0}^{L-1}\left|h_{i}[l]\right|^{2} X_{i}[k]+a_{i} \mathbf{V}_{i, i}\left(\underline{X}_{i}[k]-\underline{X}_{i}^{(0)}[k]\right)+\sum_{\substack{j=1 \\
j \neq i}}^{N} a_{j} \mathbf{U}_{i, j}\left(\underline{X}_{j}[k]-\underline{\hat{X}}_{j}^{(0)}[k]\right)+n_{i}[k]} \\
\underline{\tilde{X}}_{i}^{(m-1)}\left[k-m\left\lfloor\frac{L-1}{D}\right\rfloor\right] \triangleq\left[\tilde{X}_{i}^{(m-1)}\left[k-(m-1)\left\lfloor\frac{L-1}{D}\right\rfloor\right], \tilde{X}_{i}^{(m-1)}\left[k-(m-1)\left\lfloor\frac{L-1}{D}\right\rfloor-1\right], \ldots, \tilde{X}_{i}^{(m-1)}\left[k-(m+1)\left\lfloor\frac{L-1}{D}\right\rfloor\right]\right]^{T} \\
\underline{\hat{X}}_{i}^{(m-1)}\left[k-m\left\lfloor\frac{L-1}{D}\right\rfloor\right]=\operatorname{sgn}\left(\underline{\tilde{X}}_{i}^{(m-1)}\left[k-m\left\lfloor\frac{L-1}{D}\right\rfloor\right]\right) \\
\tilde{X}_{i}^{(m)}\left[k-m\left\lfloor\frac{L-1}{D}\right\rfloor\right]=Y_{i}\left[k-m\left\lfloor\frac{L-1}{D}\right\rfloor\right]-a_{i} \mathbf{V}_{i, i} \hat{X}_{i}^{(m-1)}\left[k-m\left\lfloor\frac{L-1}{D}\right\rfloor\right]-\sum_{\substack{j=1 \\
j \neq i}}^{N} a_{j} \mathbf{U}_{i, j} \underline{\hat{X}}_{j}^{(m-1)}\left[k-m\left\lfloor\frac{L-1}{D}\right\rfloor\right.
\end{gathered}
$$

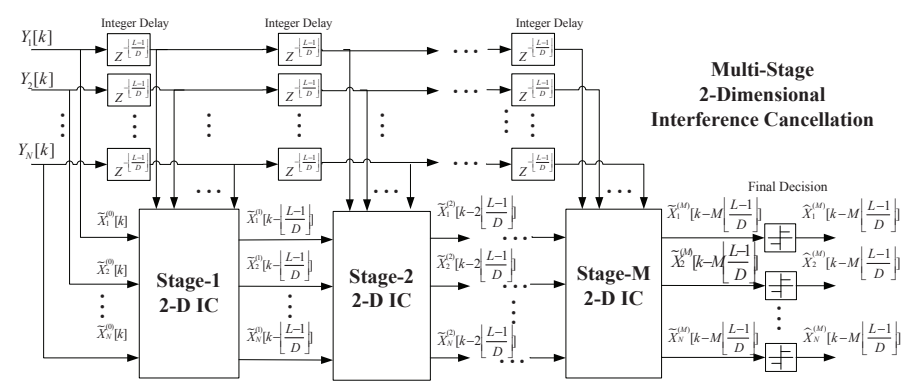

Fig. 3. The diagram of the M-stage 2D parallel interference cancellation.

the proposed 2D parallel interference cancellation. In this part, we generalize the basic single-stage scheme into a multi-stage scheme, in which the signal quality is refined after each stage of interference cancellation ${ }^{11}$.

Fig. 3 shows the diagram of an M-stage interference cancellation scheme. Basically, as the procedures proceed, in each stage, the same 2D parallel interference cancellation is performed in attempt to remove both the ISI and IUI with the updated estimates of the transmitted symbols. Each stage takes the soft-bits from the previous stage as the input, based on which tentative decisions are made to estimate the interference and generate the soft-bits of the current stage as the output.

More specifically, in the context of multiple stages, the $m$-th stage's operation can be described as follows:

- Delay and Buffering: The soft-bits from the previous stage (the $(m-1)$-th stage) are delayed and buffered to form a soft-bit vector for each user, such that for all $i \in\{1,2, \ldots, N\}, \underline{\tilde{X}}_{i}^{(m-1)}\left[k-m\left\lfloor\frac{L-1}{D}\right\rfloor\right]$ can be obtained as (20).

\footnotetext{
${ }^{11}$ This is true as long as the BER performance of the initial stage is not too high.
}

- Tentative Decision: The tentative decisions are made based on the soft-bits from the previous stage (the $(m-1)$-th stage) in attempt to estimate the transmitted symbols, i.e., for all $i \in\{1,2, \ldots, N\}$, the tentative decision vector $\underline{\hat{X}}_{i}^{(m-1)}\left[k-m\left\lfloor\frac{L-1}{D}\right\rfloor\right]$ can be obtained as (21). In $(21), \operatorname{sgn}(\cdot)$ is the sign function applied elementwise to the operand when the operand is a vector.

- Interference Cancellation: The soft-bits of the current stage (the $m$-th stage) are generated by subtracting the estimated interference terms from the original signals $\left\{Y_{i}\right\}$. The soft-bit of User $i$ generated by the $m$-th stage is given by (22).

In particular, such an $M$-stage scheme is initialized by setting the soft-bits of the initial stage (the 0-th stage) as

$$
\tilde{X}_{1}^{(0)}[k]=Y_{1}[k], \tilde{X}_{2}^{(0)}[k]=Y_{2}[k], \ldots, \tilde{X}_{N}^{(0)}[k]=Y_{N}[k] .
$$

After the signal flows through all the $M$ stages, the final decision is made for each user based on the output of the Stage- $M$ interference cancellation, for all $i \in\{1,2, \ldots, N\}$,

$$
\hat{X}_{i}^{(M)}\left[k-M\left\lfloor\frac{L-1}{D}\right\rfloor\right]=\operatorname{sgn}\left(\tilde{X}_{i}^{(M)}\left[k-M\left\lfloor\frac{L-1}{D}\right\rfloor\right]\right) .
$$

Comparing the timing of the estimated symbols after the final decision $\hat{X}_{i}^{(M)}\left[k-M\left\lfloor\frac{L-1}{D}\right\rfloor\right]$ and the original TRM output signal $Y_{i}[k]$, one can see that each stage incurs a delay of $\left\lfloor\frac{L-1}{D}\right\rfloor$. Therefore, the total detection delay grows linearly with the number of stages, but is independent of the number of users. When it comes to the complexity, the complexity of the proposed 2D parallel interference cancellation increases linearly with the number of users, as opposed to many other joint detection schemes whose complexity grows exponentially with the number of users. 


\section{Performance Analysis of The Single-stage INTERFERENCE CANCELLATION}

In this section, we analyze the estimation performance of the proposed 2-dimensional interference cancellation scheme, assuming that BPSK symbols are transmitted. As one will see, the performance analysis associated with this scheme is complicated by the fact that the tentative decisions are highly correlated in both time domain and user's signature domain, rather than independent of one another. In the following part, we focus on the single-stage 2D interference cancellation and analyze the resulting BER performance.

The theoretical analysis of the estimation error probability of the multi-stage 2D parallel interference cancellation scheme can be very difficult, due to the nonlinearity of the tentative decision and complicated correlation of the combined signals after multi-stage interference cancellation. We will use simulations to evaluate the BER performance of the multi-stage scheme.

\section{A. Statistical Model on the BER Performance}

In the proposed 2D parallel interference cancellation scheme, tentative decisions $\left\{\hat{X}_{i}^{(0)}[k]\right\}$ based on the TRM output $\left\{Y_{i}[k]\right\}$ are used to cancel the ISI and IUI in the first stage. The resulting soft-bit of User $i$ can be represented as in (19).

A hard decision (10) is made on $\tilde{X}_{i}^{(1)}[k]$ i.e. $\hat{X}_{i}^{(1)}[k]=\operatorname{sgn}\left(\tilde{X}_{i}^{(1)}[k]\right)$. To calculate the error probability $\operatorname{Pr}\left[\hat{X}_{i}^{(1)}[k] \neq X_{i}[k]\right]$, one needs a statistical model to characterize the total interference in (19). Although the BPSK symbols $\left\{X_{i}\right\}$ for all $i \in\{1,2, \ldots, N\}$ are i.i.d. and follow a very simple Bernoulli distribution, the exact distribution of the interference is much more complicated due to the large numbers of $L$ (the length of the multi-path channels) and $N$ (the number of users). On the other hand, however, for sufficiently large $L$ and $N$, it is reasonable to assume a Gaussian model for the remaining (after the cancellation) ISI and IUI.

Assuming then a Gaussian model for both the (remaining) ISI and IUI, the error probability for the single-stage cancellation is given by $P_{e r r}^{(1)}(i)=Q\left(\sqrt{S I N R_{i}^{(1)}}\right)$, where

$$
S I N R_{i}^{(1)}=\frac{\left|a_{i}\right|^{2} \sum_{l=0}^{L-1}\left|h_{i}[l]\right|^{2}}{P_{I S I}^{(1)}(i)+P_{I U I}^{(1)}(i)+\sigma_{N}^{2}} .
$$

To calculate the residual ISI power $P_{I S I}^{(1)}(i)$ and IUI power $P_{I U I}^{(1)}(i)$ in (25), we first define the error correlation matrix $R_{j, w}^{(0)}$ in (26) for $\forall j, w \in\{1,2, \ldots, N\}$ which will help simplify the notation in the following discussion.

Consequently, the ISI power for User $i$ after the first stage interference cancellation can be written as (27). Similarly, the remaining IUI for User $i$ can be represented as (28).

With (27) and (28), the calculation of the interference power for User $i$ reduces to calculate the error correlation matrix $R_{j, w}^{(0)}$, which will be addressed in the remaining part of this section.

\section{B. The Error Correlation Matrix}

The error correlation matrix $R_{j, w}^{(0)}$ defined in (26) consists of four different correlation matrices, which together characterize the correlation between the estimation errors of User $j$ and User $w$. Exact calculation of these correlation matrices can be very difficult, we approximate them using various techniques, more detailed in the following discussion.

Since the BPSK symbols are i.i.d. Bernoulli random variables over $\{-1,+1\}$, the first term $E\left[\underline{X}_{j}[k] \underline{X}_{w}^{T}[k]\right]$ (the data correlation matrix) in (26) can be calculated very easily, i.e.,

$$
E\left[\underline{X}_{j}[k] \underline{X}_{w}^{T}[k]\right]= \begin{cases}\mathbf{I}, & \text { if } j=w, \\ \underline{\mathbf{0}}, & \text { if } j \neq w .\end{cases}
$$

where $\mathbf{I}$ and $\underline{\mathbf{0}}$ are the identity matrix and the zero matrix of size $\left(2\left\lfloor\frac{L-1}{D}\right\rfloor+1\right)$-by- $\left(2\left\lfloor\frac{L-1}{D}\right\rfloor+1\right)$, respectively.

1) Cross Correlation Matrix $E\left[\underline{X}_{j}[k] \underline{\hat{X}}_{w}^{(0) T}[k]\right]$ : Define $M_{j, w}^{(0)} \triangleq E\left[\underline{X}_{j}[k] \underline{\hat{X}}_{w}^{(0) T}[k]\right]$, for $\forall w, j \in\{1,2, \ldots, N\}$. Note that the element at the $m$-th row and $n$-th column of the matrix $M_{j, w}^{(0)}$ has the following form ${ }^{12}$

$$
\left(M_{j, w}^{(0)}\right)_{(m, n)}=E\left[X_{j}[k] \hat{X}_{w}^{(0)}[k+m-n]\right],
$$

which is given in Theorem 1 .

Theorem 1. Denoting $\tau=m-n$ in (30), the expected value $E\left[X_{j}[k] \hat{X}_{w}^{(0)}[k+m-n]\right]$ is given by the following:

- when $|\tau|>\left\lfloor\frac{L-1}{D}\right\rfloor$,

$$
\left(M_{j, w}^{(0)}\right)_{(m, n)}=E\left[X_{j}[k] \hat{X}_{j}^{(0)}[k+\tau]\right]=0
$$

- when $\tau \in\left\{\tau \in \mathbb{Z}|| \tau \mid \leqslant\left\lfloor\frac{L-1}{D}\right\rfloor\right\}$, denoting $t=L-1+$ $D \tau$, then

$$
\begin{aligned}
& E\left[X_{j}[k] \hat{X}_{w}^{(0)}[k+\tau]\right] \\
= & \operatorname{sgn}\left(a_{j}\left(g_{w} * h_{j}\right)[t]\right) \operatorname{Pr}\left[\left|\Psi_{j, w}[\tau]\right| \leqslant\left|a_{j}\left(g_{w} * h_{j}\right)[t]\right|\right] \\
\approx & \operatorname{sgn}\left(a_{j}\left(g_{w} * h_{j}\right)[t]\right)\left(1-2 Q\left(\frac{\left|a_{j}\left(g_{w} * h_{j}\right)[t]\right|}{\sigma_{\Psi_{j, w}[\tau]}}\right)\right)
\end{aligned}
$$

Proof: Please refer to Appendix I.

2) Estimation Correlation Matrix $E\left[\underline{\hat{X}}_{j}^{(0)}[k] \underline{\hat{X}}_{w}^{(0) T}[k]\right]$ : Define $C_{j, w}^{(0)} \triangleq E\left[\underline{\hat{X}}_{j}^{(0)}[k] \underline{\hat{X}}_{w}^{(0) T}[k]\right]$, for $\forall j, w \in 1,2, \ldots, N$. Denoting $\tau=m-n$, the element on the $m$-th row and $n$ th column of the matrix $\left(C_{j, w}^{(0)}\right)$ can be then represented as follows

$$
\begin{aligned}
& \left(C_{j, w}^{(0)}\right)_{m, n} \\
= & E\left[\operatorname{sgn}\left(Y_{j}[k] Y_{w}[k+\tau]\right)\right] \\
= & \operatorname{Pr}\left[Y_{j}[k] Y_{w}[k+\tau] \geqslant 0\right]-\operatorname{Pr}\left[Y_{j}[k] Y_{w}[k+\tau]<0\right] \\
= & 2 \operatorname{Pr}\left[Y_{j}[k] Y_{w}[k+\tau] \geqslant 0\right]-1,
\end{aligned}
$$

\footnotetext{
${ }^{12}$ Note that for the value of any element $\left(M_{j, w}^{(0)}\right)_{(m, n)}$ is a function of $m-n$, independent of the specific value of $k$.
} 


$$
\begin{gathered}
R_{j, w}^{(0)} \triangleq E\left[\left(\underline{X}_{j}[k]-\underline{\hat{X}}_{j}^{(0)}[k]\right)\left(\underline{X}_{w}[k]-\underline{\hat{X}}_{w}^{(0)}[k]\right)^{T}\right] \\
=E\left[\underline{X}_{j}[k] \underline{X}_{w}^{T}[k]\right]-E\left[\underline{X}_{j}[k] \underline{\hat{X}}_{w}^{(0) T}[k]\right]-\left(E\left[\underline{X}_{w}[k] \underline{\hat{X}}_{j}^{(0) T}[k]\right]\right)^{T}+E\left[\underline{\hat{X}}_{j}^{(0)}[k] \underline{\hat{X}}_{w}^{(0) T}[k]\right] \\
P_{I S I}^{(1)}(i)=a_{i}^{2} E\left[\mathbf{V}_{i, i}\left(\underline{X}_{i}[k]-\underline{\hat{X}}_{i}^{(0)}[k]\right)\left(\underline{X}_{i}[k]-\underline{\hat{X}}_{i}^{(0)}[k]\right)^{T} \mathbf{V}_{i, i}^{T}\right]=a_{i}^{2} \mathbf{V}_{i, i} R_{i, i}^{(0)} \mathbf{V}_{i, i}^{T}, \\
P_{I U I}^{(1)}(i)=\sum_{\substack{j=1 \\
j \neq i}}^{N} \sum_{\substack{w=1 \\
w \neq i}}^{N} E\left[a_{j} a_{w} \mathbf{U}_{i, j}\left(\underline{X}_{j}[k]-\underline{\hat{X}}_{j}^{(0)}[k]\right)\left(\underline{X}_{w}[k]-\underline{\hat{X}}_{w}^{(0)}[k]\right)^{T} \mathbf{U}_{i, w}^{T}\right]=\sum_{\substack{j=1 \\
j \neq i}}^{N} \sum_{\substack{w=1 \\
w \neq i}}^{N} a_{j} a_{w} \mathbf{U}_{i, j} R_{j, w}^{(0)} \mathbf{U}_{i, w}^{T}
\end{gathered}
$$

which can approximated by modeling $\left(Y_{j}[k], Y_{w}[k+\tau]\right)$ as bivariate Gaussian random variables in Theorem (2).

Theorem 2. By modeling $\left(Y_{j}[k], Y_{w}[k+\tau]\right)$ as bivariate Gaussian random variables, the probability $\operatorname{Pr}\left[Y_{j}[k] Y_{w}[k+\right.$ $\tau] \geqslant 0]$ in (33) can be given by the following

$$
\begin{aligned}
& \operatorname{Pr}\left[Y_{j}[k] \geqslant 0, Y_{w}[k+\tau] \geqslant 0\right] \\
= & \operatorname{Pr}\left[Y_{j}[k] \leqslant 0, Y_{w}[k+\tau] \leqslant 0\right] \\
= & \frac{1}{4}+\frac{\arcsin \left(\rho_{j, w}(\tau)\right)}{2 \pi},
\end{aligned}
$$

where

$$
\begin{gathered}
\rho_{j, w}(\tau)=\frac{E\left[Y_{j}[k] Y_{w}[k+\tau]\right]}{\sigma_{1} \sigma_{2}}, \\
\sigma_{1}^{2}=E\left[Y_{j}^{2}[k]\right]=\sum_{d=1}^{N} a_{d}^{2} \mathbf{U}_{j, d} \mathbf{U}_{j, d}^{T}+\sigma_{N}^{2}, \\
\sigma_{2}^{2}=E\left[Y_{w}^{2}[k+\tau]\right]=\sum_{d=1}^{N} a_{d}^{2} \mathbf{U}_{w, d} \mathbf{U}_{w, d}^{T}+\sigma_{N}^{2} .
\end{gathered}
$$

Proof: Please refer to Appendix II for the proof of this theorem and the calculation of $E\left[Y_{j}[k] Y_{w}[k+\tau]\right]$.

Based on Theorem 2, for any element $\left(C_{j, w}^{(0)}\right)_{m, n}$ in the matrix $\left(C_{j, w}^{(0)}\right)$, it holds that

$$
\begin{aligned}
\left(C_{j, w}^{(0)}\right)_{m, n} & =2 \operatorname{Pr}\left[Y_{j}[k] Y_{w}[k+\tau] \geqslant 0\right]-1 \\
& =\frac{2}{\pi} \arcsin \left(\rho_{j, w}(\tau)\right) .
\end{aligned}
$$

To summarize, we have derived an analytical approximation for each of the four components of the error correlation matrix in (26). Therefore, for any given $j, w \in\{1,2, \ldots, N\}$, it holds that

$$
R_{j, w}^{(0)} \approx\left\{\begin{array}{cc}
\mathbf{I}-M_{j, j}^{(0)}-\left(M_{j, j}^{(0)}\right)^{T}+C_{j, j}^{(0)} & \text { if } j=w, \\
-M_{j, w}^{(0)}-\left(M_{w, j}^{(0)}\right)^{T}+C_{j, w}^{(0)} & \text { if } j \neq w,
\end{array}\right.
$$

so that both the residual ISI power (27) and IUI power (28) can be calculated using (39). Then for any given user of interest (for instance, User $i$ ), the post-cancellation $\operatorname{SINR} S I N R_{i}^{(0)}$ (25) can be obtained to compute its BER performance.
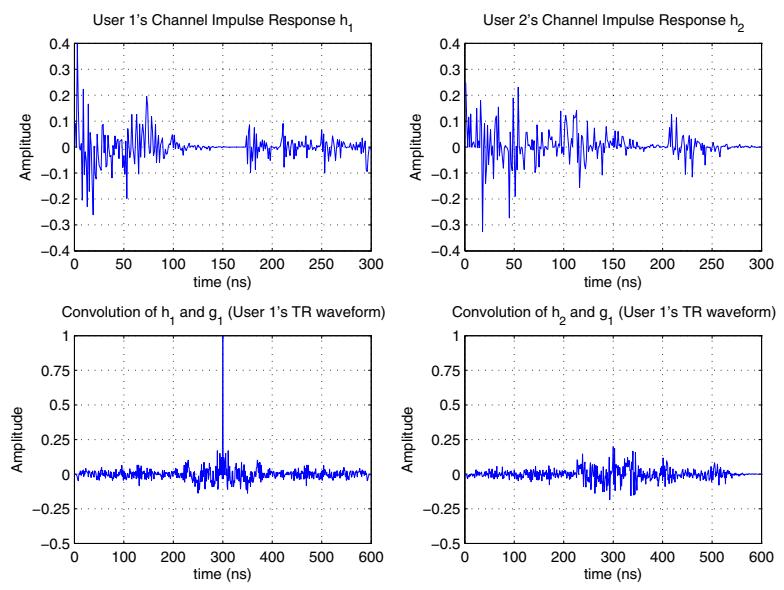

Fig. 4. Examples of IEEE 802.15.4a outdoor NLOS channels

\section{Simulation Results}

In this section, we present some simulation results on the BER performance of the proposed 2D parallel interference cancellation scheme. To study the proposed scheme in a relatively realistic setting, we used the IEEE 802.15.4a (UWB) outdoor non-line-of-sight (NLOS) channel model ${ }^{13}$ [22] to evaluate the BER performance of the proposed scheme.

Fig. 4 shows an example of two typical channel impulse responses under such a channel model and their convolutions with the TR signature waveforms. The channels shown in Fig. 4 are randomly generated according to the channel model specified in [22], with the system sampling period $T_{s}=1$ ns and the channel length truncated ${ }^{14}$ at $L T_{s}=300$ ns (i.e., $\mathrm{L}=300$ ). In Fig. 4, the convolution between User 1's CIR $h_{1}$ and its matched TR signature waveform $g_{1}$ exhibits a prominent central peak at $\left(h_{1} * g_{1}\right)[L-1]$, demonstrating the temporal focusing effect of the TR technique; on the other hand, the amplitude of the convolution between the TR signature waveform $g_{1}$ and the mismatched CIR $h_{2}$ is significantly smaller than the central peak $\left(h_{1} * g_{1}\right)[L-1]$, demonstrating the virtual spatial focusing effect in the user's signature domain.

In the following part of this section, we present our numer-

\footnotetext{
${ }^{13}$ In such a channel model, each channel tap is a real number.

${ }^{14}$ This is because the amplitude of the remaining paths after $300 \mathrm{~ns}$ is typically small enough to be neglected
} 


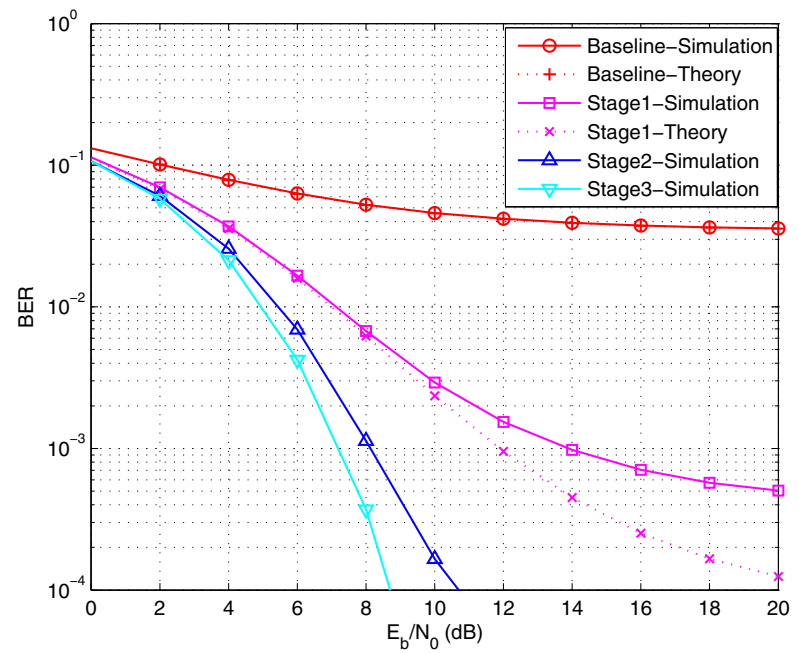

Fig. 5. The BER performance of the $\mathrm{D}=16 \mathrm{~N}=5$.

ical evaluation on the BER performance of the proposed 2D interference cancellation scheme. In our simulation, the CIRs for different users are randomly and independently generated using the IEEE 802.15.4a channel model, with $T_{s}=1 \mathrm{~ns}$ and $L=300$. Without loss of generality, the CIR of each user is normalized so that $\sum_{k=0}^{L-1}\left|h_{i}[k]\right|^{2}=1, \quad \forall i \in\{1,2, \ldots, N\}$, and assume that all the power control coefficients $a_{i}=$ $1, \quad \forall i \in\{1,2, \ldots, N\}^{15}$. A large number of independent trials of channel realizations were conducted and averaged to characterize the average performance of the proposed scheme under this channel model.

\section{A. BER vs $E_{b} / N_{0}$}

In this part, we show the BER performance versus $E_{b} / N_{0}$ (the energy-per-bit to noise-power-spectral-density ratio) with various combinations of rate back-off factor $D$ and the total number of users $N$. The energy-per-bit $E_{b}$ is normalized to 1 by the assumption that each BPSK symbol $X_{i}[k] \in\{-1,+1\}$ has a unit power; accordingly, the power of the received AWGN $\tilde{n}[k]$ at the BS is then given by

$$
\sigma_{N}^{2}=\frac{N_{0}}{2}=\left(\frac{2 E_{b}}{N_{0}}\right)^{-1} .
$$

Fig. 5 and Fig. 6 show the BER performances of the proposed 2D parallel interference cancellation scheme (up to 3 stages), and compare with the basic TRDMA system without interference cancellation. Fig. 5 considers that case where there are $N=5$ end-users accessing the BS at the same time with a rate back-off factor $D=16$ (about $5.3 \%$ of the channel length $L=300$ ); Fig. 6 then presents the BER performance with $N=10$ and $D=32$ (about $10.7 \%$ of the channel length).

From both figures, significant BER performance gain is achieved by the proposed 2D parallel interference cancellation scheme, compared with the baseline TRDMA system without

\footnotetext{
${ }^{15}$ To implement an equal power allocation among the users.
}

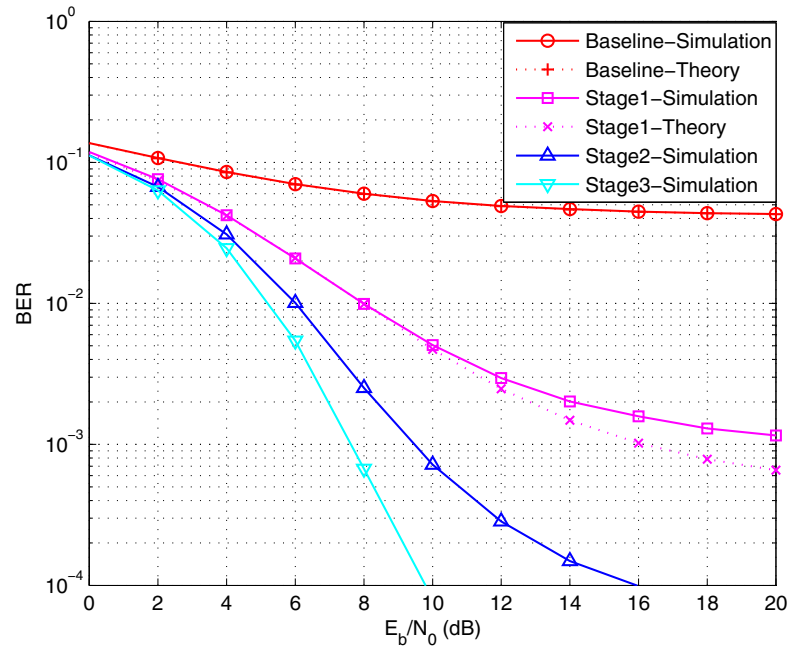

Fig. 6. The BER performance of the $\mathrm{D}=32 \mathrm{~N}=10$.

interference cancellation. Additional gain is achieved by cascading more stages of the proposed interference cancellation scheme, at the price of increased decoding delay which grows linearly with the total number of stages. The largest gainper-stage is obtained by the first stage, with the marginal gain diminishes for the following stages. Therefore, there always exists such a tradeoff between the system performance and the number of stages, which should be determined by the applications. Lastly, the theoretical approximation derived in Section V matches reasonably well with the simulation results, considering its complicated correlation between tentative decisions and the Gaussian assumptions we made in the approximation. Comparing Fig. 5 and Fig. 6, one can see that the accuracy of this approximation improves as the number of users increases, which agrees with the central limit theorem.

\section{B. Further discussion on high SNR regime}

In high SNR regime, the TRDMA system becomes an interference limited system. In this part, to evaluate the effectiveness of the proposed 2D interference cancellation scheme, we show how the system performs in the high SNR regime. In the following numerical results, the $E_{b} / N_{0}$ is chosen as 15 $\mathrm{dB}$ to represent high SNR.

1) BER vs the number of users $N$ : Fig. 7 shows how the BER performance changes with the number of users $N$ with high enough SNR. As shown in the example of Fig. 7 with the rate back-off factor $D=32$, the BER increases with the number of users $N$ due to the increased IUI. Targeting at a certain BER level (e.g $10^{-3}$ ), the proposed 2D interference cancellation enables more users to transmit simultaneously and therefore increases the system capacity. The benefit of using the interference cancellation diminishes when the BER of the baseline system (considered as the initial stage) is above a certain threshold. This is because of the fact that the interference cancellation scheme at the receiver eventually relies on the tentative decisions of the detected symbols to cancel the interference, whose effectiveness inevitably depends on the quality of those tentative decisions. 


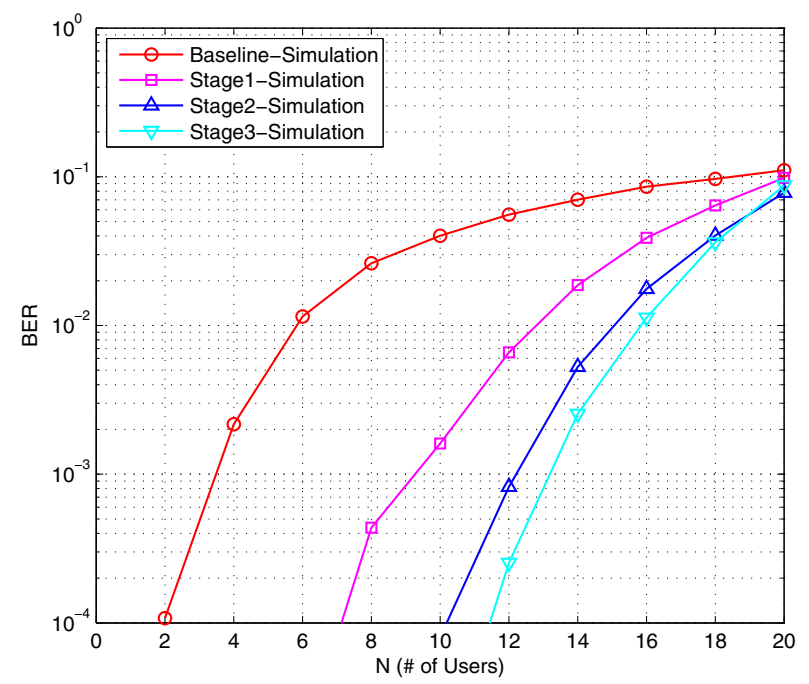

Fig. 7. The BER performance vs number of users $N$.

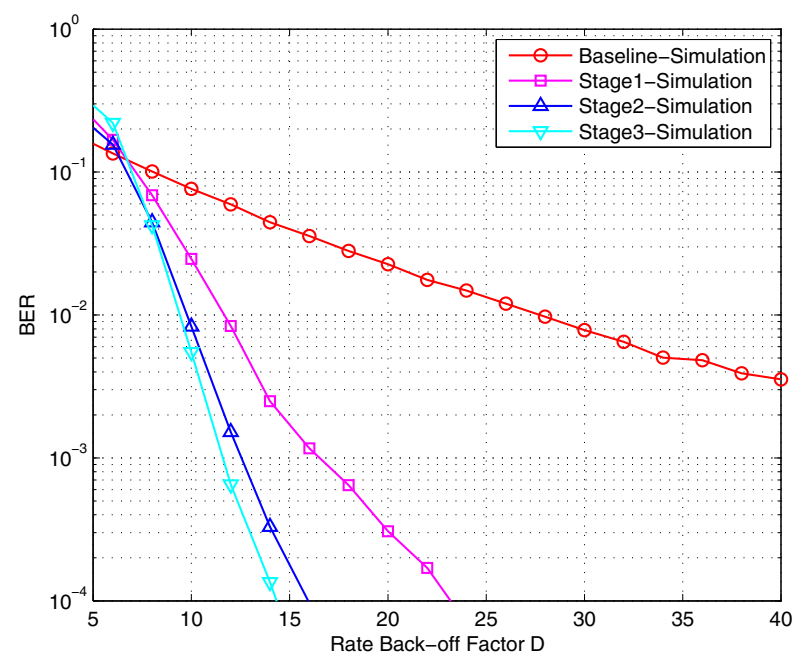

Fig. 8. The BER performance vs rate back-off factor $D$.

2) BER vs the rate back-off factor D: Fig. 8 shows how the BER performance is affected by the rate back-off factor $D$ with high enough SNR, assuming that there are 5 users accessing the BS simultaneously. Since both the ISI and the IUI are reduced with a larger rate back-off factor $D$, the BER decreases as $D$ increases. From Fig. 8, one can see that thanks to the proposed 2D parallel interference cancellation scheme, the TRDMA system could use a smaller $D$ to achieve the same BER, which translates to higher throughput for each user. Finally, it is also worth noting that when $D$ is very small (e.g., for $D=6$ (only $2 \%$ of the channel length) in Fig. 8), there can be more erroneous tentative decisions (i.e. very high BER $\left(>10^{-1}\right)$ for the initial stage) which in turn actually enhance the interference power, rather than canceling. However, most practical TRDMA applications operate at a much lower BER level ${ }^{16}$ for the proposed interference cancellation scheme to contribute.

\footnotetext{
${ }^{16}$ A lower BER level can always be achieved by increasing the rate back-off factor $D$ and/or reducing the number of concurrent users $N$.
}

\section{CONCLUSION}

In this paper, we introduced a multi-user TRDMA uplink architecture and proposed a 2D parallel interference cancellation scheme to enhance the system performance. As we discussed in this paper, such a TRDMA uplink architecture keeps the cost of end-users at a minimum level, and reuses the processing power at the base station (BS) that has already been made available for the downlink. The proposed 2D parallel interference cancellation scheme utilizes the tentative decisions of detected symbols to effectively cancel the interference in both the time dimension (ISI) and the user dimension (IUI). To further improve the BER performance, we extended our discussion to a multi-stage scheme by cascading multiple stages of the proposed 2D interference cancellation, with a total delay that increases linearly with the number of stages, but independent of the number of users. Theoretical analysis was made on the BER performance of the single-stage cancellation, and the approximated theoretical result well matched the simulation results. The performance results demonstrated that the proposed 2D parallel interference cancellation scheme can significantly improve the system performance of the TRDMA system.

\section{APPENDIX I:PROOF OF THEOREM 1}

When $|\tau|>\left\lfloor\frac{L-1}{D}\right\rfloor, X_{j}[k]$ and $\hat{X}_{w}^{(0)}[k+\tau]=$ $\operatorname{sgn}\left(Y_{w}[k+\tau]\right)$ are independent (as can be seen in (7)) and therefore

$$
\left(M_{j, w}^{(0)}\right)_{(m, n)}=E\left[X_{j}[k] \hat{X}_{j}^{(0)}[k+\tau]\right]=0 .
$$

For any given number $\tau \in\left\{\tau \in \mathbb{Z}|| \tau \mid \leqslant\left\lfloor\frac{L-1}{D}\right\rfloor\right\}$, we have (40).

According to (7), for $-\left\lfloor\frac{L-1}{D}\right\rfloor \leqslant \tau \leqslant\left\lfloor\frac{L-1}{D}\right\rfloor$, the signal $Y_{w}[k+\tau]-a_{j}\left(g_{w} * h_{j}\right)[L-1+D \tau] X_{j}[k]$ is independent of $X_{j}[k]$. We define $\Psi_{j, w}[\tau] \triangleq Y_{w}[k+\tau]-a_{j}\left(g_{w} * h_{j}\right)[L-$ $1+D \tau] X_{j}[k]$, which is a linear combination of a large number of independent variables. By central limit theorem, we approximate $\Psi_{j, w}[\tau]$ as a Gaussian random variable with zero mean and the variance shown as follows

$\sigma_{\Psi_{j, w}[\tau]}^{2}=\sigma_{N}^{2}+\sum_{v=1}^{N} a_{v}^{2} \mathbf{U}_{w, v} \mathbf{U}_{w, v}^{T}-a_{j}^{2}\left|\left(g_{w} * h_{j}\right)[L-1+D \tau]\right|^{2}$

Consequently, (40) can be further calculated as (42).

\section{APPENDIX II: PROOF OF THEOREM 2}

To approximate the probability $\operatorname{Pr}\left[Y_{j}[k] Y_{w}[k+\tau] \geqslant 0\right]$, we model $\left(Y_{j}[k], Y_{w}[k+\tau]\right)$ as bivariate Gaussian random variables, following the multidimensional central limit theorem [20]. It is easy to show that both $Y_{j}[k]$ and $Y_{w}[k+\tau]$ have zero mean, i.e.

$$
E\left[Y_{j}[k]\right]=E\left[Y_{w}[k+\tau]\right]=0 .
$$

The covariance matrix $\Sigma=\left(\begin{array}{cc}\sigma_{1}^{2} & \rho_{j, w}(\tau) \sigma_{1} \sigma_{2} \\ \rho_{j, w}(\tau) \sigma_{1} \sigma_{2} & \sigma_{2}^{2}\end{array}\right)$ can be given by the following:

$$
\sigma_{1}^{2}=E\left[Y_{j}^{2}[k]\right]=\sum_{d=1}^{N} a_{d}^{2} \mathbf{U}_{j, d} \mathbf{U}_{j, d}^{T}+\sigma_{N}^{2}
$$




$$
\begin{aligned}
\left(M_{j, w}^{(0)}\right)_{(m, n)}= & E\left[X_{j}[k] \hat{X}_{w}^{(0)}[k+\tau]\right] \\
= & \frac{1}{2}\left[E\left[\hat{X}_{w}^{(0)}[k+\tau] \mid X_{j}[k]=1\right]-E\left[\hat{X}_{w}^{(0)}[k+\tau] \mid X_{j}[k]=-1\right]\right] \\
= & \frac{1}{2}\left[\operatorname{Pr}\left[Y_{w}[k+\tau] \geqslant 0 \mid X_{j}[k]=1\right]-\operatorname{Pr}\left[Y_{w}[k+\tau]<0 \mid X_{j}[k]=1\right]\right]- \\
& \frac{1}{2}\left[\operatorname{Pr}\left[Y_{w}[k+\tau] \geqslant 0 \mid X_{j}[k]=-1\right]-\operatorname{Pr}\left[Y_{w}[k+\tau]<0 \mid X_{j}[k]=-1\right]\right]
\end{aligned}
$$

$$
\begin{aligned}
& E\left[X_{j}[k] \hat{X}_{w}^{(0)}[k+\tau]\right] \\
= & \frac{1}{2}\left[\operatorname{Pr}\left[\Psi_{j, w}[\tau] \geqslant-a_{j}\left(g_{w} * h_{j}\right)[L-1+D \tau]\right]-\operatorname{Pr}\left[\Psi_{j, w}[\tau]<-a_{j}\left(g_{w} * h_{j}\right)[L-1+D \tau]\right]\right]- \\
& \frac{1}{2}\left[\operatorname{Pr}\left[\Psi_{j, w}[\tau] \geqslant a_{j}\left(g_{w} * h_{j}\right)[L-1+D \tau]\right]-\operatorname{Pr}\left[\Psi_{j, w}[\tau]<a_{j}\left(g_{w} * h_{j}\right)[L-1+D \tau]\right]\right] \\
= & \operatorname{sgn}\left(a_{j}\left(g_{w} * h_{j}\right)[L-1+D \tau]\right) \operatorname{Pr}\left[\left|\Psi_{j, w}[\tau]\right| \leqslant\left|a_{j}\left(g_{w} * h_{j}\right)[L-1+D \tau]\right|\right] \\
\approx & \operatorname{sgn}\left(a_{j}\left(g_{w} * h_{j}\right)[L-1+D \tau]\right)\left(1-2 Q\left(\frac{\left|a_{j}\left(g_{w} * h_{j}\right)[L-1+D \tau]\right|}{\sigma_{\Psi_{j, w}}[\tau]}\right)\right)
\end{aligned}
$$

$$
\begin{gathered}
\sigma_{2}^{2}=E\left[Y_{w}^{2}[k+\tau]\right]=\sum_{d=1}^{N} a_{d}^{2} \mathbf{U}_{w, d} \mathbf{U}_{w, d}^{T}+\sigma_{N}^{2} \\
\rho_{j, w}(\tau)=\frac{E\left[Y_{j}[k] Y_{w}[k+\tau]\right]}{\sigma_{1} \sigma_{2}}
\end{gathered}
$$

where $E\left[Y_{j}[k] Y_{w}[k+\tau]\right]$ is given by (47).

In (47), the expectation $\Lambda[\tau] \triangleq E\left[\underline{X}_{d}[k] \underline{X}_{d}^{T}[k+\tau]\right]$ is a $\left(2\left\lfloor\frac{L-1}{D}\right\rfloor+1\right)$-by- $\left(2\left\lfloor\frac{L-1}{D}\right\rfloor+1\right)$ square Toeplitz matrix with its elements determined by $(\Lambda[\tau])_{p, q}=\lambda_{q-p}$, where the value of $\lambda_{q-p}$ is given as follows:

$$
\lambda_{q-p}= \begin{cases}1, & \text { if } \quad q-p=\tau, \\ 0, & \text { if } \quad q-p \neq \tau .\end{cases}
$$

Thanks to the special structure of $\Lambda[\tau]$, the value of $\mathbf{U}_{j, d}(\Lambda[\tau]) \mathbf{U}_{w, d}^{T}$ can be represented by a simple function $\Pi\left(\mathbf{U}_{j, d}, \mathbf{U}_{w, d}, \tau\right)$ defined in (49). In (49), $\operatorname{dim}(\cdot)$ equals to the dimensionality of the operand vector.

For the second term $\underline{g}_{j} E\left[\underline{\tilde{n}}[D k] \underline{\tilde{n}}^{T}[D(k+\tau)]\right] \underline{g}_{w}^{T}$ in (47), one could have a similar expression using the definition of the function $\Pi$ in (49)

$$
\begin{aligned}
& \underline{g}_{j} E\left[\underline{\tilde{n}}[D k] \underline{\tilde{n}}^{T}[D(k+\tau)]\right] \underline{g}_{w}^{T} \\
= & \left\{\begin{array}{cll}
\sigma_{N}^{2} \Pi\left(\underline{g}_{j}, \underline{g}_{w}, D \tau\right), & \text { if } & |\tau| \leqslant\left\lfloor\frac{L-1}{D}\right\rfloor, \\
0 & \text { if } & |\tau|>\left\lfloor\frac{L-1}{D}\right\rfloor .
\end{array}\right.
\end{aligned}
$$

Combining the results of (49) and (50), the cross correlation between $Y_{j}[k]$ and $Y_{w}[k+\tau]$ is given by

$$
\begin{aligned}
& E\left[Y_{j}[k] Y_{w}[k+\tau]\right] \\
& \left\{\begin{array}{cc}
\sigma_{N}^{2} \Pi\left(\underline{g}_{j}, \underline{g}_{w}, D \tau\right)+\sum_{d=1}^{N} a_{d}^{2} \Pi\left(\mathbf{U}_{j, d}, \mathbf{U}_{w, d}, \tau\right), & \text { if }|\tau| \leqslant\left\lfloor\frac{L-1}{D}\right\rfloor, \\
\sum_{d=1}^{N} a_{d}^{2} \Pi\left(\mathbf{U}_{j, d}, \mathbf{U}_{w, d}, \tau\right), & \text { if }|\tau|>\left\lfloor\frac{L-1}{D}\right\rfloor .
\end{array}\right.
\end{aligned}
$$

which can be substituted into (46) to obtain the value of $\rho_{j, w}(\tau)$.

Upon obtaining the probability distribution of $\left(Y_{j}[k], Y_{w}[k+\tau]\right)$ by assuming a bivariate normal distribution model, the quadrant probability is readily and analytically given by [21]

$$
\begin{aligned}
& \operatorname{Pr}\left[Y_{j}[k] \geqslant 0, Y_{w}[k+\tau] \geqslant 0\right] \\
= & \operatorname{Pr}\left[Y_{j}[k] \leqslant 0, Y_{w}[k+\tau] \leqslant 0\right] \\
= & \frac{1}{4}+\frac{\arcsin \left(\rho_{j, w}(\tau)\right)}{2 \pi} .
\end{aligned}
$$

\section{REFERENCES}

[1] F. Han, Y. Yang, B. Wang, Y. Wu, and K. J. R. Liu, "Time-reversal division multiple access over multi-path channels," IEEE Trans. Commun., vol. 60, no. 7, pp. 1953-1965, July 2012.

[2] J. G. Proakis, Digital Communications, $4^{\text {th }}$ ed. McGraw-Hill, 2001.

[3] G. L. Stuber, Principles of Mobile Communications, $2^{\text {nd }}$ ed. Kluwer, 2001.

[4] A. J. Goldsmith, Wireless Communication. Cambridge University Press, 2005.

[5] D. Tse and P. Viswanath, Fundamental of Wireless Communication. Cambridge University Press, 2005.

[6] B. Wang, Y. Wu, F. Han, Y. H. Yang, and K. J. R. Liu, "Green wireless communications: a time-reversal paradigm," IEEE J. Sel. Areas Commun., vol 29, no 8, pp. 1698-1710, Sep. 2011.

[7] M. Lienard, P. Degauque, V. Degardin, and I. Vin, "Focusing gain model of time-reversed signals in dense multipath channels," IEEE Antennas and Wireless Propag. Lett., vol. 11, pp. 1064-1067, 2012.

[8] Y. Chen, Y. Yang, F. Han, and K. J. R. Liu, "Time-reversal wideband communications,' IEEE Signal Process. Lett., vol. 20, no. 12, pp. 12191222, Dec. 2013.

[9] M. Fink, C. Prada, F. Wu, and D. Cassereau, "Self focusing in inhomogeneous media with time reversal acoustic mirrors," in Proc. 1989 IEEE Ultrasonics Symposium, vol. 1, pp. 681-686.

[10] C. Prada, F. Wu, and M. Fink, "The iterative time reversal mirror: a solution to self-focusing in the pulse echo mode," J. Acoustic Society of America, vol. 90, pp. 1119-1129, 1991.

[11] Y. Yang, B. Wang, W. Lin, and K. J. R. Liu, "Near-optimal waveform design for sum rate optimization in time-reversal multiuser downlink systems," IEEE Trans. Wireless Commun., vol. 12, no. 1, pp. 346-357, Jan 2013. 


$$
\begin{aligned}
E\left[Y_{j}[k] Y_{w}[k+\tau]\right] & =E\left[\left(\sum_{d=1}^{N} a_{d} \mathbf{U}_{j, d} \underline{X}_{d}[k]+n_{j}[k]\right)\left(\sum_{d=1}^{N} a_{v} \mathbf{U}_{w, v} \underline{X}_{v}[k+\tau]+n_{w}[k+\tau]\right)\right] \\
& =\sum_{d=1}^{N} \sum_{v=1}^{N} a_{d} a_{v} \mathbf{U}_{j, d} E\left[\underline{X}_{d}[k] \underline{X}_{v}^{T}[k+\tau]\right] \mathbf{U}_{w, v}^{T}+E\left[n_{j}[k] n_{w}[k+\tau]\right] \\
& =\sum_{d=1}^{N} a_{d}^{2} \mathbf{U}_{j, d} E\left[\underline{X}_{d}[k] \underline{X}_{d}^{T}[k+\tau]\right] \mathbf{U}_{w, d}^{T}+\underline{g}_{j} E\left[\underline{\tilde{n}}[D k] \underline{\tilde{n}}^{T}[D(k+\tau)]\right] \underline{g}_{w}^{T}
\end{aligned}
$$

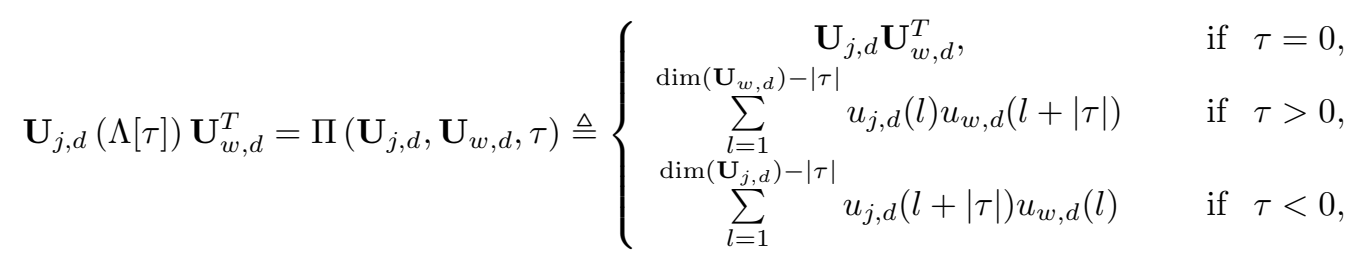

[12] S. Verdu, "Minimum probability of error for asynchronous Gaussian multiple-access channels," IEEE Trans. Inf. Theory, vol. IT-32, pp. 8596, Jan. 1986.

[13] S. Verdu, "Optimum multiuser asymptotic efficiency," IEEE Trans. Commun., vol. COM-34, pp. 890-897, Sep. 1986.

[14] M. K. Varanasi and B. Aazhang, "Multistage detection in asynchronous code-division multiple-access communications," IEEE Trans. Commun., vol. 38, no. 4, pp. 509-519, Apr. 1990.

[15] A. J. Viterbi, "Very low rate convolutional codes for maximum theoretical performance of spread-spectrum multiple-access channels," IEEE Trans. Sel. Areas Commun., vol. 8, no. 4, pp. 641-649, May 1990.

[16] D. Divsalar and M. K. Simon, "Improved CDMA performance using parallel interference cancellation," JPL Publication, 95-21, Oct. 1995.

[17] A. Duel-Hallen, J. Holtzman, and Z. Zvonar, "Multiuser detection for CDMA systems," IEEE Personal Commun., pp. 46-58, Apr. 1995.

[18] S. Moshavi, "Multi-user detection for DS-CDMA communications," IEEE Commun. Mag., pp. 124-136, Oct. 1996.

[19] D. Divsalar, M. K. Simon, and D. Raphaeli, "Improved parallel interference cancellation for CDMA," IEEE Trans. Commun., vol. 46, no. 2, Feb. 1998.

[20] A. W. van der Vaart, Asymptotic Statistics. Cambridge University Press, 1998.

[21] C. Rose and M. D. Smith, "The multivariate normal distribution," Mathematica J., vol. 6 no. 1, pp. 32-37, Winter 1996.

[22] A. F. Molisch et al., "IEEE 802.15.4a channel model-final report," Tech. Rep. Document IEEE 802.15-04-0662-02-004a, 2005.

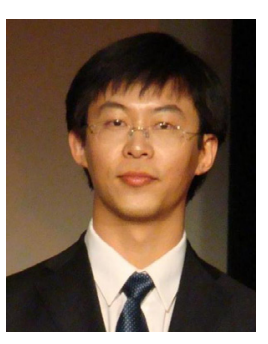

Feng Han (S'08-M'14) received the B.S. and M.S. degrees from Tsinghua University, Beijing, China, in 2007 and 2009, respectively; and the Ph.D. degree from University of Maryland, College Park, MD, USA, in 2013, all in Electrical Engineering. Currently, he is a senior engineer with Corporate Research and Development, Qualcomm Inc., San Diego, CA. His research interests include wireless communications and networking, game theory, signal processing and communication theory.

$\mathrm{He}$ is a recipient of the first prize in the 19th Chinese Mathematical Olympiad, the Best Thesis Award of Tsinghua University, the honor of Excellent Graduate of Tsinghua University, the A. James Clark School of Engineering Distinguished Graduate Fellowship in 2009 and the Future Faculty Fellowship in 2012, both from the University of Maryland, College Park. His work on time reversal technique was recognized by the university-level Invention of the Year Award and The Jimmy H. C. Lin Invention Award, both at University of Maryland in 2013, and his work on MIMO system received a Best Paper Award for at IEEE WCNC'08.

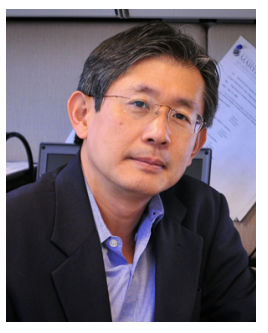

K. J. Ray Liu (F'03) was named a Distinguished Scholar-Teacher of University of Maryland, College Park, in 2007, where he is Christine Kim Eminent Professor of Information Technology. He leads the Maryland Signals and Information Group conducting research encompassing broad areas of signal processing and communications with recent focus on cooperative and cognitive communications, social learning and network science, information forensics and security, and green information and communications technology.

Dr. Liu is the recipient of numerous honors and awards including IEEE Signal Processing Society Technical Achievement Award and Distinguished Lecturer. He also received various teaching and research recognitions from University of Maryland including university-level Invention of the Year Award; and Poole and Kent Senior Faculty Teaching Award, Outstanding Faculty Research Award, and Outstanding Faculty Service Award, all from A. James Clark School of Engineering. An ISI Highly Cited Author, Dr. Liu is a Fellow of IEEE and AAAS.

Dr. Liu is Past President of IEEE Signal Processing Society where he has served as Vice President - Publications and Board of Governor. He was the Editor-in-Chief of IEEE Signal Processing Magazine and the founding Editorin-Chief of EURASIP Journal on Advances in Signal Processing. 